\title{
Dust formation history of galaxies: A critical role of metallicity* for the dust mass growth by accreting materials in the interstellar medium
}

\author{
Ryosuke S. Asano ${ }^{1}$, Tsutomu T. Takeuchi ${ }^{1}$, Hiroyuki Hirashita ${ }^{2}$, and Akio K. Inoue ${ }^{3}$ \\ ${ }^{1}$ Department of Particle and Astrophysical Science, Nagoya University, Furo-cho, Chikusa-ku, Nagoya 464-8602, Japan \\ ${ }^{2}$ Academia Sinica Institute for Astronomy and Astrophysics, P. O. Box 23-141, Taipei 10617, Taiwan \\ ${ }^{3}$ College of General Education, Osaka Sangyo University, 3-1-1 Nakagaito, Daito, Osaka 574-8530, Japan
}

(Received November 7, 2011; Revised April 22, 2012; Accepted April 27, 2012; Online published March 12, 2013)

\begin{abstract}
This paper investigates the main driver of dust mass growth in the interstellar medium (ISM) by using a chemical evolution model of a galaxy with metals (elements heavier than helium) in the dust phase, in addition to the total amount of metals. We consider asymptotic giant branch (AGB) stars, type II supernovae (SNe II), and dust mass growth in the ISM, as the sources of dust, and SN shocks as the destruction mechanism of dust. Furthermore, to describe the dust evolution precisely, our model takes into account the age and metallicity (the ratio of metal mass to ISM mass) dependence of the sources of dust. We have particularly focused on the dust mass growth, and found that in the ISM this is regulated by the metallicity. To quantify this aspect, we introduce a "critical metallicity", which is the metallicity at which the contribution of stars (AGB stars and SNe II) equals that of the dust mass growth in the ISM. If the star-formation timescale is shorter, the value of the critical metallicity is higher, but the galactic age at which the metallicity reaches the critical metallicity is shorter. From observations, it was expected that the dust mass growth was the dominant source of dust in the Milky Way and dusty QSOs at high redshifts. By introducing a critical metallicity, it is clearly shown that the dust mass growth is the main source of dust in such galaxies with various star-formation timescales and ages. The dust mass growth in the ISM is regulated by metallicity, and we emphasize that the critical metallicity serves as an indicator to judge whether the grain growth in the ISM is the dominant source of dust in a galaxy, especially because of the strong, and nonlinear, dependence on the metallicity.
\end{abstract}

Key words: Dust, extinction, galaxies: infrared, galaxies: evolution, galaxies: starburst, stars: formation.

\section{Introduction}

Stellar light, in particular at shorter wavelengths, is absorbed by dust and re-emitted as a far-infrared thermal emission from the dust (e.g., Witt and Gordon, 2000, and references therein). Therefore, dust affects the spectral energy distributions of galaxies (e.g., Takagi et al., 1999; Granato et al., 2000; Noll et al., 2009; Popescu et al., 2011). The existence of dust in galaxies also affects the star-formation activity. Dust grains increase the molecularformation rate by two orders of magnitude compared to the case without dust (e.g., Hollenbach and McKee, 1979), and the interstellar medium (ISM) is cooled efficiently by molecules and dust. Consequently, star formation is activated drastically by dust. Hence, dust is one of the most important factors for the evolution of galaxies (e.g., Hirashita and Ferrara, 2002; Yamasawa et al., 2011).

The amount of dust in galaxies is one of the crucial factors to interpret the observational information of galaxies, since dust exists ubiquitously and the radiation from stars is always affected by dust attenuation. However, in spite of

*The ratio of metal (elements heavier than helium) mass to ISM mass.

Copyright (C) The Society of Geomagnetism and Earth, Planetary and Space Sciences (SGEPSS); The Seismological Society of Japan; The Volcanological Society of Japan; The Geodetic Society of Japan; The Japanese Society for Planetary Sciences; TERRAPUB

doi:10.5047/eps.2012.04.014 its importance, the evolution of the amount of dust has not been completely established yet. There are some key factors involved in understanding the dust evolution of galaxies. One of these is the ratio of the metal (elements heavier than helium) mass to the ISM mass, which is referred to as the "metallicity". Since dust grains consist of metals, it is natural to think that the evolution of dust is closely related to metallicity. In general, galaxies are believed to evolve from a state with a very low metallicity and a very small amount of dust to one with higher amounts of metal and dust. Hence, it is mandatory to model the formation and evolution of dust grains in galaxies along with the evolution of metallicity (e.g., Dwek and Scalo, 1980; Hirashita, 1999a, b; Inoue, 2003; Yamasawa et al., 2011).

Dust grains are formed by the condensation of metals. A significant part of the metals released by stellar mass loss during stellar evolution, or supernovae $(\mathrm{SNe})$ at the end of the life of stars, condense into dust grains. Dust grains not only originate from stars, but are also destroyed by SNe blast waves (e.g., Jones et al., 1994, 1996; Nozawa et al., 2003; Zhukovska et al., 2008). In addition, we should consider the dust mass growth in the ISM by the accretion of atoms and molecules of refractory elements onto grains (e.g., Liffman and Clayton, 1989; Dwek, 1998; Draine, 2009; Jones and Nuth, 2011).

What kind of dust formation processes are dominant at each stage of galaxy evolution is a very important question 
for understanding the evolution history of the ISM and star formation in galaxies. However, since dust evolution depends strongly on the age and metallicity of a galaxy, it is not an easy question to answer. Up to now, dust evolution has been studied with various models. For example, in young galaxies, $\mathrm{SNe}$ have been considered as the source of dust because they are the final stage of massive stars whose lifetime is short, and asymptotic giant branch (AGB) stars have been neglected because of their longer lifetime. However, Valiante et al. (2009) showed that the AGB stars also contribute to the dust production in young galaxies, and cannot be neglected even on a short timescale of $~ 500 \mathrm{Myr}$. A more elaborate survey of the parameter space for the dust formation by SNe and AGB stars has been carried out by Gall et al. (2011a). They showed that the contribution of AGB stars exceeds that of SNe II, at several $100 \mathrm{Myr}$, if the ratio between the metal and dust mass produced by SNe II is less $\sim 0.01$ and mass-heavy IMF with a mass range 1$100 \mathrm{M}_{\odot}$.

As for the dust mass growth, the ISM is considered to be the main source of dust in various galaxies. For example, the present dust amount observed in the Milky Way cannot be explained if the source of dust was only stars, suggesting that we must consider the dust mass growth in the ISM in evolved galaxies (e.g., Liffman and Clayton, 1989; Dwek, 1998; Draine, 2009; Jones and Nuth, 2011). Recently, dusty quasars (total dust mass $>10^{8} \mathrm{M}_{\odot}$ ) have been discovered at high redshifts (e.g., Beelen et al., 2006; Wang et al., 2008), and theoretical studies on dust sources at high redshifts are currently carried out actively (e.g., Michałowski et al., 2010b; Gall et al., 2011a, b; Pipino et al., 2011; Valiante et al., 2011). These have shown that it is difficult to explain the total dust amount in these QSOs only with stellar contributions, and the importance of dust mass growth in the ISM has been discussed. The next question is what controls the point where the dust mass growth in the ISM dominates the total dust mass production in galaxies. Therefore, although each physical process has already been extensively discussed, there emerges the crucial question: what kind of dust production process is dominant at each stage of galaxy evolution? And, in particular, when does dust mass growth become dominant as a source of dust mass?

The central aim of this work is to address this question. In this paper, we investigate what is the main driver of dust mass growth in the ISM. Since all sources of dust production are tightly related to each other in dust evolution, it is crucial to treat these processes in a unified framework to understand the evolution of dust in galaxies. Here, we adopt a model based on a chemical evolution model in a same manner as Hirashita (1999b), Calura et al. (2008), and Inoue (2011). This is because their models consider the main dust production/destruction processes that affect the dust evolution of galaxies, which makes it easy to compare our results to previous ones. From this work, we find that the dust mass growth in the ISM is regulated by a critical metallicity, the details of which are described in Subsection 3.2. Although dust mass growth can occur at any time during the age of a galaxy, there is a moment at which the dust mass growth becomes greater than the contribution from other sources of dust.
This paper is organized as follows. In Section 2, we describe the model developed for this work. In Section 3, we describe, and discuss, the basic results obtained by our model. The main topic of this paper, critical metallicity, is introduced and extensively examined in Subsection 3.2. Section 4 is devoted to the conclusions. The solar metallicity is set to be $Z_{\odot}=0.02$ (Anders and Grevesse, 1989) throughout this paper.

\section{Dust Evolution Model of Galaxies}

In this section, we describe a simple chemical evolution model with dust which examines what determines the point where the dust mass growth in the ISM becomes dominant. The dust evolution model is developed in the same manner as in Hirashita (1999b) and Inoue (2011).

\subsection{Equations of galaxy evolution}

In this section, we describe the equations of the mass evolution of stars and the ISM which contains metal and dust in galaxies. We use a simple one-zone model, because we are interested in the global properties of galaxies. Also, we assume a closed-box model. Thus, the total baryon mass $M_{\text {tot }}$ (the sum of the stellar mass and the ISM mass) is a constant. However, since $M_{\text {tot }}$ is just a scale factor in our model, this value does not affect the physical properties of galaxies nonlinearly.

In this work, we do not consider the effects of inflow and outflow. However, they may not influence the properties of dust and metal enrichment in galaxies for the following reasons: An inflow makes not only the metallicity, but also the dust-to-gas mass ratio, small, because usually an inflow is considered to be metal- and dust-poor. As for an outflow, it expels ISM components (gas, metal and dust) out of a galaxy. However, if all ISM components flow out together, the metallicity and the dust-to-gas mass ratio do not change.

Under these parameters, the equations of the time evolution of the total stellar mass $M_{*}$, ISM mass $M_{\mathrm{ISM}}$, metal mass $M_{Z}$, and dust mass $M_{\mathrm{d}}$ are (e.g., Lisenfeld and Ferrara, 1998; Hirashita, 1999b)

$$
\begin{aligned}
\frac{\mathrm{d} M_{*}(t)}{\mathrm{d} t}= & \operatorname{SFR}(t)-R(t), \\
\frac{\mathrm{d} M_{\mathrm{ISM}}(t)}{\mathrm{d} t}= & -\operatorname{SFR}(t)+R(t), \\
\frac{\mathrm{d} M_{Z}(t)}{\mathrm{d} t}= & -Z(t) \operatorname{SFR}(t)+R_{Z}(t)+Y_{Z}(t), \\
\frac{\mathrm{d} M_{\mathrm{d}}(t)}{\mathrm{d} t}= & -\mathcal{D}(t) \operatorname{SFR}(t)+Y_{\mathrm{d}}(t)-\frac{M_{\mathrm{d}}}{\tau_{\mathrm{SN}}} \\
& +\eta \frac{M_{\mathrm{d}}(1-\delta)}{\tau_{\mathrm{acc}}},
\end{aligned}
$$

where SFR is the star-formation rate, $Z(t) \equiv M_{\mathrm{Z}} / M_{\mathrm{ISM}}$ is the metallicity, $\mathcal{D} \equiv M_{\mathrm{d}} / M_{\mathrm{ISM}}$ is the dust-to-gas mass ratio, $\delta \equiv M_{\mathrm{d}} / M_{Z}$ is the fraction of metals in dust. $\eta$ is the mass fraction of cold clouds where the accretion process occurs, $\tau_{\mathrm{SN}}$ and $\tau_{\mathrm{acc}}$ are the timescales of dust destruction and accretion, respectively. The definitions of these timescales are described later. Also, $R(t)$ and $R_{Z}(t)$ are the total baryon mass returned by stars and the total metal mass once injected in stars and just returned in the ISM per unit time, respectively. $Y_{Z}(t)$ and $Y_{\mathrm{d}}(t)$ are the total metal mass 
newly produced and ejected by stars, and the total dust mass ejected by stars per unit time, respectively. There is another notation where $Y_{Z}$ includes $R_{Z}$ (e.g., Inoue, 2011). In this case, the value of $Y_{Z}$ is different from that in this paper because of the different definition.

For the SFR, we adopt the Schmidt law (Schmidt, 1959); SFR $\propto M_{\mathrm{ISM}}^{n}$. Here, we adopt $n=1$ for simplicity. Thus, the SFR is expressed as

$$
\operatorname{SFR}(t)=\frac{M_{\mathrm{ISM}}(t)}{\tau_{\mathrm{SF}}},
$$

where $\tau_{\mathrm{SF}}$ is the star-formation timescale.

Also, $R(t), R_{Z}(t), Y_{Z}(t)$ and $Y_{\mathrm{d}}(t)$ are written by

$$
\begin{aligned}
R(t)= & \int_{m_{\text {cut }}(t)}^{100 \mathrm{M}_{\odot}}\left[m-\omega\left(m, Z\left(t-\tau_{m}\right)\right)\right] \\
& \times \phi(m) \operatorname{SFR}\left(t-\tau_{m}\right) \mathrm{d} m, \\
R_{Z}(t)= & \int_{m_{\text {cut }}(t)}^{100 \mathrm{M}_{\odot}}\left[m-\omega\left(m, Z\left(t-\tau_{m}\right)\right)\right] \\
& \times \phi(m) \operatorname{SFR}\left(t-\tau_{m}\right) Z\left(t-\tau_{m}\right) \mathrm{d} m, \\
Y_{Z}(t)= & \int_{m_{\text {cut }}(t)}^{100 \mathrm{M}_{\odot}} m_{Z}\left(m, Z\left(t-\tau_{m}\right)\right) \\
& \times \phi(m) \operatorname{SFR}\left(t-\tau_{m}\right) \mathrm{d} m, \\
Y_{\mathrm{d}}(t)= & \int_{m_{\text {cut }}(t)}^{100 \mathrm{M}_{\odot}} m_{\mathrm{d}}\left(m, Z\left(t-\tau_{m}\right)\right) \\
& \times \phi(m) \operatorname{SFR}\left(t-\tau_{m}\right) \mathrm{d} m,
\end{aligned}
$$

where $\phi(m)$ is the initial mass function (IMF), $\omega(m, Z(t)), m_{Z}(m, Z(t))$ and $m_{\mathrm{d}}(m, Z(t))$ are the remnant mass, metal mass newly produced and ejected, and dust mass produced and ejected, by a star of initial mass $m$ and metallicity $Z$, respectively. The lifetime of a star of initial mass $m$, expressed as $\tau_{m}$, is taken from Raiteri et al. (1996). In this work, since its metallicity dependence is weak, we always take the lifetime of the case of the solar metallicity. The lower limit $m_{\text {cut }}(t)$ is the mass of a star with a lifetime $\tau_{m}=t$. As for the IMF, we adopt the Larson IMF (Larson, 1998) in the stellar mass range 0.1-100 $\mathrm{M}_{\odot}$,

$$
\phi(m) \propto m^{-(\alpha+1)} \exp \left(-\frac{m_{\mathrm{ch}}}{m}\right) .
$$

Here, we take $\alpha=1.35$ and $m_{\mathrm{ch}}=0.35 \mathrm{M}_{\odot}$. Also, we normalize it as

$$
\int_{0.1 \mathrm{M}_{\odot}}^{100 \mathrm{M}_{\odot}} m \phi(m) \mathrm{d} m=1 .
$$

To calculate Eqs. (6)-(9), we take the data of remnant mass $(\omega(m, Z))$, metal mass $\left(m_{Z}(m, Z)\right)$ and dust mass $\left(m_{\mathrm{d}}(m, Z)\right)$ of stars, with a mass $m$ and metallicity $Z$, from some previous works.

In this work, we consider AGB stars and SNe II as stellar sources, but we neglect the SNe Ia for simplicity. Nozawa et al. (2011) recently proved that SNe Ia produce little dust. Furthermore, Calura et al. (2008) have shown that the dust destruction rate by $\mathrm{SNe}$ Ia is about $1 / 10$ of that by SNe II. As for the metals ejected by SNe Ia, they play an important role in the chemical evolution of galaxies (e.g., Matteucci et $a l ., 2009)$. However, since we do not discuss the abundance ratio of each metal but, rather, the total metallicity, we do not take into account the contribution of SNe Ia.

In this paper, we assume that the mass ranges of AGB stars and $\mathrm{SNe}$ II are 1-8 $\mathrm{M}_{\odot}$ and 8-40 $\mathrm{M}_{\odot}$, respectively. Also, we assume that all stars with initial masses $m>40$ $\mathrm{M}_{\odot}$ evolve to black holes without SN explosions (Heger et al., 2003).

As for the remnant and metal masses, the data are taken from van den Hoek and Groenewegen (1997) for AGB stars with a mass range $1-7 \mathrm{M}_{\odot}$ and metallicities $Z=\left(5.0 \times 10^{-2}, 0.2,0.4,1.0\right) Z_{\odot}$, and from Woosley and Weaver (1995) for SNe II with a mass range $12-40 \mathrm{M}_{\odot}$ and metallicities $Z=\left(5.0 \times 10^{-2}, 0.1,1.0\right) Z_{\odot}$. As for the dust mass, the data is taken from Zhukovska et al. (2008) for AGB stars with a mass range $1-7 \mathrm{M}_{\odot}$ and metallicities $Z=\left(5.0 \times 10^{-2}, 0.1,0.2,0.4,0.75,1.0\right) \mathrm{Z}_{\odot}$, and from Valiante et al. (2009) for SNe II with a mass range 12-40 $\mathrm{M}_{\odot}$ and metallicities $Z=\left(5.0 \times 10^{-2}, 1.0\right) \mathrm{Z}_{\odot}$, which are quoted from Bianchi and Schneider (2007).

Although stardust yields are not completely understood, theoretical predictions of SNe II recently show a good agreement with observations of nearby supernova remnants (SNRs) (e.g., Nozawa et al., 2010). We considered the current model based on these latest results. However, some problems still remain unsolved (e.g., nucleation efficiency). As for the dust yield of AGB stars, we take similar stardust yields to those of Valiante et al. $(2009,2011)$ and Gall et al. (2011a) whilst their yields may be uncertain. However, we note that after the dust mass growth in the ISM becomes dominant, the dust abundance is insensitive to stardust yields (Inoue, 2011). Thus, although there exist slight uncertainties in dust yields, we can discuss the activation mechanism for dust mass growth in the ISM without ambiguity.

\subsection{Dust destruction timescale}

It is thought that $\mathrm{SNe}$ are the main source of dust destruction. This dust destruction process depends on various parameters (density and temperature of the ISM, the explosion energy of the $\mathrm{SNe}$, etc.), and is very complex (e.g., Jones et al., 1994, 1996; Nozawa et al., 2006). In this work, we adopt the equations presented by Mckee (1989).

The timescale of dust destruction $\tau_{\mathrm{SN}}$ is expressed as

$$
\tau_{\mathrm{SN}}=\frac{M_{\mathrm{ISM}}(t)}{\epsilon m_{\mathrm{swept}} \gamma_{\mathrm{SN}}(t)},
$$

where $\epsilon$ is the efficiency of the dust destruction by SN shocks, and is defined as the ratio of the destroyed dust to the total swept dust by SN shocks, $m_{\text {swept }}$ is the swept ISM mass by a $\mathrm{SN}$ shock, $\gamma_{\mathrm{SN}}(t)$ is the $\mathrm{SN}$ rate. In this work, we assume $\epsilon=0.1$ (Mckee, 1989; Nozawa et al., 2006).

The $\mathrm{SN}$ rate $\gamma(t)$ is expressed as

$$
\gamma_{\mathrm{SN}}(t)=\int_{m_{\text {cut }}(t)>8 \mathrm{M}_{\odot}}^{40 \mathrm{M}_{\odot}} \phi(m) \operatorname{SFR}\left(t-\tau_{m}\right) \mathrm{d} m .
$$

The range of the integration is the mass range where the $\mathrm{SNe}$ can occur (Heger et al., 2003). So, if $t<\tau_{40 \mathrm{M}_{\odot}}$, $\gamma_{\mathrm{SN}}(t)=0.0$. 
The swept ISM mass by SN shocks, $m_{\text {swept }}$, depends on both the density and metallicity of the ISM. In the case of a higher density, since the amount of materials which block the SN blast wave is larger, the swept mass becomes smaller. Further, the line cooling by metals is more efficient in the ISM of a higher metallicity and, as a result, the swept mass becomes smaller. To consider these effects, we adopt the fitting formula derived by Yamasawa et al. (2011)

$$
m_{\text {swept }}=1535 n_{\mathrm{SN}}^{-0.202}\left[\left(Z / \mathrm{Z}_{\odot}\right)+0.039\right]^{-0.289}\left[\mathrm{M}_{\odot}\right],
$$

where $n_{\mathrm{SN}}$ is the ISM density around $\mathrm{SNe}$, and we assume $n_{\mathrm{SN}}=1.0 \mathrm{~cm}^{-3}$ as a representative value.

\subsection{Metal accretion timescale}

Dust mass in galaxies increases as a result of not only supply from stars but also accretion of refractory elements onto pre-existing dust in clouds (e.g., Liffman and Clayton, 1989; Draine, 2009; Jones and Nuth, 2011). This accretion process is called "dust mass growth". Here, we use the term "clouds", which stands for the cool component in the ISM. In our study, we neglect volatile dusts. Although, indeed, they exist in clouds, if clouds disappear or the temperature increases, such dusts evaporate. A more precise treatment will be developed in future work. The dust mass growth rate in clouds can be expressed as (e.g., Hirashita, 2000; Inoue, 2003, 2011)

$$
\left(\frac{\mathrm{d} M_{\mathrm{d}}}{\mathrm{d} t}\right)_{\mathrm{acc}}=\eta N \pi\left\langle a^{2}\right\rangle \alpha \rho_{Z}^{\mathrm{gas}}\langle v\rangle,
$$

where $\eta$ is the mass fraction of the clouds, $N$ is the number of dust grains, $\left\langle a^{2}\right\rangle$ is the 2nd moment of a grain size $a, \alpha$ is the mean sticking coefficient of metals, $\rho_{Z}^{\text {gas }}$ is the mass density of gaseous metals that are not contained in the dust, and $\langle v\rangle$ is the mean velocity of metals in the gas phase. Since we assume a spherical dust grain for simplicity, we have

$$
m_{\mathrm{d}}=\frac{4 \pi\left\langle a^{3}\right\rangle \sigma}{3}
$$

so

$$
N=\frac{M_{\mathrm{d}}}{m_{\mathrm{d}}}=\frac{3 M_{\mathrm{d}}}{4 \pi\left\langle a^{3}\right\rangle \sigma},
$$

where $m_{\mathrm{d}}$ is the mean mass of the grain, $\left\langle a^{3}\right\rangle$ is the $3 \mathrm{rd}$ moment of a grain size, and $\sigma$ is the mass density of solid matter within the grains. Also,

$$
\rho_{Z}^{\mathrm{gas}}=\rho_{\mathrm{ISM}}^{\mathrm{eff}} Z(1-\delta)
$$

where $\rho_{\text {ISM }}^{\text {eff }}$ is the averaged mass density of the interstellar clouds where the accretion process occurs, and $\delta$ is the dust abundance in the metal mass. Thus, defining the accretion timescale as

$$
\tau_{\mathrm{acc}}=\frac{4\left\langle a^{3}\right\rangle \sigma}{3\left\langle a^{2}\right\rangle \alpha \rho_{\mathrm{ISM}}^{\mathrm{eff}} Z\langle v\rangle},
$$

we obtain the fourth term in the right-hand side of Eq. (4). Also, the mass density $\rho_{\text {ISM }}^{\text {eff }}$ is estimated in terms of the hydrogen number density, $n_{\mathrm{H}}$, as $\rho_{\mathrm{ISM}}^{\mathrm{eff}}=\mu m_{\mathrm{H}} n_{\mathrm{H}}$, where $\mu$ is the mean atomic weight (we assume $\mu=1.4$; i.e. the ratio of the number of a hydrogen atom and a helium atom is $10: 1$ in clouds), and $m_{\mathrm{H}}$ is the mass of a hydrogen atom.

We assume that $\alpha=1.0$ (i.e. if a molecule/atom collides with a dust grain, it always sticks to it) and $\sigma=3 \mathrm{~g} \mathrm{~cm}^{-3}$ (silicate). Considering that the dust mass growth depends on the volume-to-surface ratio of the grains, we obtain

$$
\begin{aligned}
\tau_{\mathrm{acc}} \approx & 2.0 \times 10^{7} \\
& \times\left(\frac{\bar{a}}{0.1 \mu \mathrm{m}}\right)\left(\frac{n_{\mathrm{H}}}{100 \mathrm{~cm}^{-3}}\right)^{-1} \\
& \times\left(\frac{T}{50 \mathrm{~K}}\right)^{-\frac{1}{2}}\left(\frac{Z}{0.02}\right)^{-1}[\mathrm{yr}] \\
= & \tau_{\mathrm{acc}, 0} Z^{-1},
\end{aligned}
$$

where $\tau_{\mathrm{acc}, 0}=4.0 \times 10^{5} \mathrm{yr}$, and we adopted $\bar{a}=0.1 \mu \mathrm{m}$, $n_{\mathrm{H}}=100 \mathrm{~cm}^{-3}$, and $T=50 \mathrm{~K}^{1}$. The typical size of grains $\bar{a}$ is defined as $\left\langle a^{3}\right\rangle /\left\langle a^{2}\right\rangle$ in Hirashita and Kuo (2011).

As mentioned above, we conservatively adopt $\bar{a}=0.1$ $\mu \mathrm{m}$ as a fiducial value (e.g., Inoue, 2011). Small grains may be depleted by coagulation in molecular clouds (Hirashita and Yan, 2009), which strengthens the importance of large grains. The importance of large grains is further enhanced given that the grain size distribution tends to be biased towards a large $(a \sim 0.1 \mu \mathrm{m})$ size by the destruction within SN remnants (Nozawa et al., 2007). Thus, we assume $\bar{a} \sim 0.1 \mu \mathrm{m}$ to estimate the dust mass growth timescale. Although we basically adopt $\bar{a}=0.1 \mu \mathrm{m}$, we also examine $\bar{a}=0.01 \mu \mathrm{m}$ for a quick growth case, later. Indeed, the MRN grain size distribution (Mathis et al., 1977) has $\bar{a}=0.01 \mu \mathrm{m}$ (Hirashita and Kuo, 2011). In reality, the grain size distribution in galaxies changes with time due to some processes (e.g., SN shocks, accretion, etc.). As for the contribution of the evolution of the grain size distribution, this is discussed in a paper in preparation (Asano et al., 2012).

In this paper, we consider only $\eta=0$ (no accretion growth), or 1 , in order to avoid any fine-tuning. In fact, the effect of a different choice of $\eta$ can be offset by a different choice of $n_{\mathrm{H}}$ and $T$. This allows us to merge the uncertainties of $\eta, n_{\mathrm{H}}$, and $T$ into the value of $\tau_{\mathrm{acc}, 0}$. We set $\tau_{\text {acc, } 0}=4.0 \times 10^{5} \mathrm{yr}$ as a fiducial value (e.g., Inoue, 2011). Other choices of $\tau_{\mathrm{acc}, 0}$ result in a different timing of the growth activation. This is explicitly expressed in Eq. (27) later.

\section{What Drives Dust Mass Growth in the ISM?}

In this section, we investigate what determines the point where the grain growth in the ISM dominates the total dust mass production in galaxies.

\subsection{Contribution of each physical process to the total dust mass in galaxies}

To examine when the dust mass growth becomes dominant as the main source of dust in galaxies, we first compare

${ }^{1}$ This temperature corresponds to $\langle v\rangle=0.14 \mathrm{~km} \mathrm{~s}^{-1}$. We assume $A m_{\mathrm{H}}\langle v\rangle^{2}=k T$ and adopt $A=20\left(A m_{\mathrm{H}}\right.$ is the mean mass of the colliding atoms) (Spitzer, 1978). 

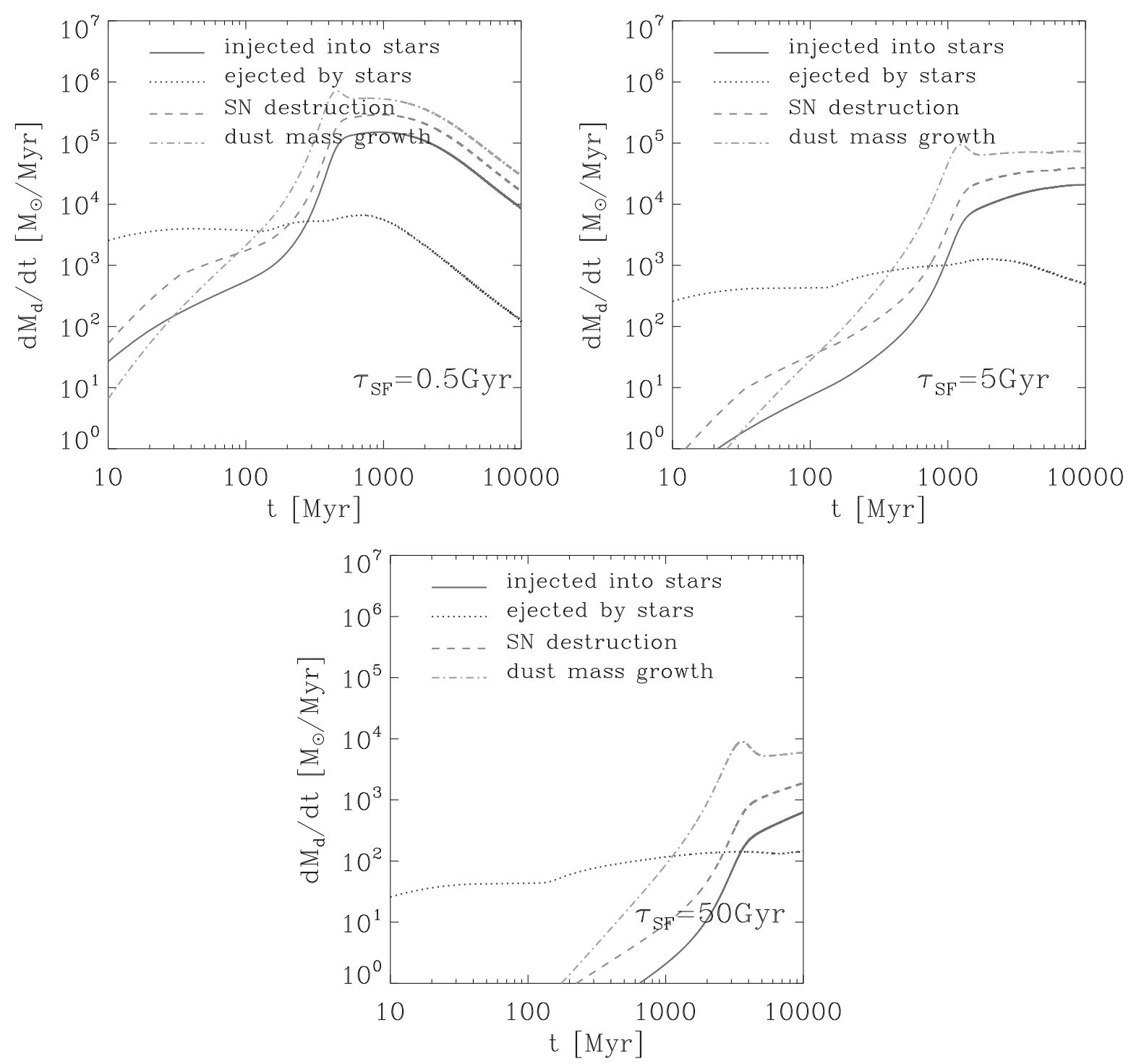

Fig. 1. Time evolution of each dust production/destruction rate (terms in the right-hand side of Eq. (4)) with $M_{\text {tot }}=10^{10} \mathrm{M}_{\odot}$ and $\eta=1.00$. The star-formation timescales are set to be $0.5 \mathrm{Gyr}$ (top-left panel), $5 \mathrm{Gyr}$ (top-right panel) and 50 Gyr (bottom panel). Solid, dotted, dashed and dot-dashed lines represent the rates of dust injection into stars, dust ejection by stars, dust destruction by SN shocks and dust mass growth in a galaxy, respectively.

each process of dust production. In Fig. 1, we show the contributions of stars (solid and dotted lines), dust destruction (dashed line) and the dust mass growth (dot-dashed line) to the total dust mass in a galaxy. Solid, dotted, dashed and dot-dashed lines represents the contributions of the 1st, 2nd, 3rd and 4th terms in the right-hand side of Eq. (4), respectively. The total baryon mass $M_{\text {tot }}$ (the sum of the total mass of stars and ISM in a galaxy) and $\eta$ are $10^{10} \mathrm{M}_{\odot}$ and 1.00 , respectively. However, as mentioned in Subsection 2.1, since $M_{\text {tot }}$ is just a scale factor, $M_{\text {tot }}$ just changes the values of the contributions of these processes linearly.

From these figures, we find that although the ejected-bystars contribution is the biggest in the early stages, as time passes, the main contributor to the dust production becomes the dust mass growth in the ISM at a given point referred to as the "switching point". For example, Liang and Li (2009) pointed out that dust produced by SNe II predominates the dust budget in galaxies in a high- $z$ Universe $(z>5)$ using the extinction curves of GRB host galaxies at high red- shifts. Their results are in good agreement with our work. Furthermore, the process of dust mass growth is expected to explain the dust amount in the Milky Way or dusty QSOs at high redshifts (e.g., Zhukovska et al., 2008; Draine, 2009; Michałowski et al., 2010a; Valiante et al., 2011). So, what determines the switching point? We will discuss this in the next section (this is the main topic in this paper).

After dust mass growth has taken place, the effect of dust destruction by $\mathrm{SN}$ shocks approaches that of dust mass growth. Thus, after dust mass growth becomes efficient, the dust amount in galaxies determines the balance between the effect of dust destruction and that of dust mass growth in the ISM (see also Inoue, 2011).

In addition, we also observe that the increase of the contribution of the dust mass growth (dot-dashed line) has a peak, after which, the increase slows down. In other words, the dust mass growth becomes less significant. We consider the reason for this. In Fig. 2, we show the time evolution of $\delta\left(=M_{Z} / M_{\mathrm{d}}\right)$ with various star-formation timescales. We 


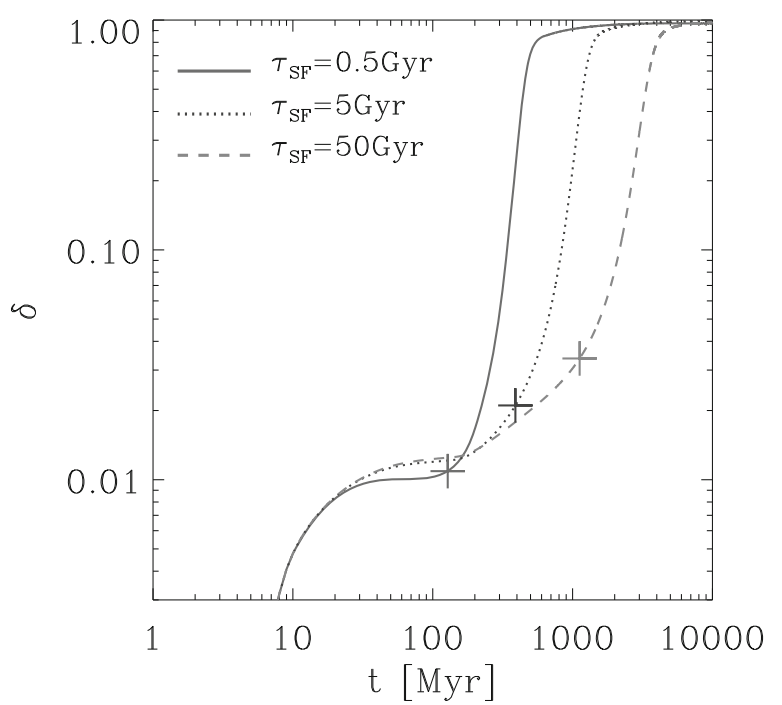

Fig. 2. Time evolution of the fraction of metals in dust, $\delta$ with $\eta=1.00$. Solid, dotted and dashed lines represent $\tau_{\mathrm{SF}}=0.5,5,50 \mathrm{Gyr}$, respectively. Cross symbols mark the switching point for each $\tau_{\mathrm{SF}}$.

find that after the value of $\delta$ increases rapidly, the value becomes constant. Hence, after the dust mass growth becomes significant, most of the metals form dust. Thus, the reason why the dust mass growth becomes less significant is the depletion of metals.

As shown in Fig. 2, the values of $\delta$ for all $\tau_{\mathrm{SF}}$ s converge to $\sim 1$. In contrast, the value for the Milky Way is about 0.5 . However, since it can be adjusted by adopting a different $\eta$, we do not try to fine-tune the convergence value of $\delta$ in this study. Inoue (2011) showed that the convergence value of $\delta$ is determined by the balance between the contribution of dust destruction by SN shocks and that of the dust mass growth (for details, the product of $\tau_{\mathrm{acc}, 0}$ and $\epsilon m_{\text {swept }}$ ).

\subsection{Critical metallicity for dust mass growth}

In this section, we introduce the main topic of this paper, the critical metallicity. This is the metallicity at the switching point (see Subsection 3.1). In our model, the sources of dust are stars (AGB stars and SNe II) and the dust mass growth in the ISM.

In order to determine the critical metallicity, we compare the second term with the fourth term of the right-hand side of Eq. (4). First, we consider the second term. From Eq. (9), if $D$ is defined as

$$
\int_{m_{\text {cut }}(t)}^{100 \mathrm{M}_{\odot}} m_{\mathrm{d}}\left(m, Z\left(t-\tau_{m}\right)\right) \phi(m) \mathrm{d} m \equiv D,
$$

then Eq. (9), with Eq. (5), can be approximated as

$$
Y_{\mathrm{d}} \simeq D \frac{M_{\mathrm{ISM}}}{\tau_{\mathrm{SF}}} .
$$

This equation is exact if SFR is constant.

Next, we consider the fourth term of the right-hand side of Eq. (4). Since $\tau_{\mathrm{acc}}=\tau_{\mathrm{acc}, 0} Z^{-1}$ and $M_{\mathrm{d}}=\delta M_{Z}=$ $\delta Z M_{\mathrm{ISM}}$, the dust mass growth term is

$$
\eta \frac{M_{\mathrm{d}}(1-\delta)}{\tau_{\mathrm{acc}}}=\frac{\eta \delta(1-\delta) Z^{2} M_{\mathrm{ISM}}}{\tau_{\mathrm{acc}, 0}} .
$$

From Eqs. (22) and (23), the metallicity at which the increasing rate of dust mass due to dust mass growth exceeds the dust production rate by stars is then presented as follows:

$$
Z=\left[\frac{D}{\eta \delta(1-\delta)}\right]^{\frac{1}{2}}\left(\frac{\tau_{\mathrm{acc}, 0}}{\tau_{\mathrm{SF}}}\right)^{\frac{1}{2}}
$$

Thus, if the metallicity of a galaxy is larger than the above metallicity, we should consider the effect of dust mass growth in the galaxy. Here, we refer to the metallicity as the critical metallicity $Z_{\mathrm{cr}}$, which is the metallicity at the switching point. To obtain the value of $Z_{\mathrm{cr}}$, hereafter, we adopt $\delta=0.02$ and $D=5 \times 10^{-4}$. As for the value of $\delta$, from Fig. 2, the value of $\delta$ is in the range of $0.01-0.04$ at the switching point for each $\tau_{\mathrm{SF}}$. Furthermore, although $\delta$ is dependent on time, before the dust mass growth becomes effective to the total dust mass, $\delta$ is determined only by the contribution of stars (see Appendix A). This contribution is in the range 0.01-0.04 in our calculation (Fig. A.1 in Appendix A). Thus, we take $\delta=0.02$ as a representative value. Also, since we found from numerical calculation that the range of $D$ is $10^{-4}$ to $10^{-3}$, we take $D=5 \times 10^{-4}$ as a representative value.

For reasons of clarification, we compare our discussion with a similar work by Inoue (2011). Inoue (2011) defined a critical metallicity to compare the contribution of dust destruction by SN shocks with that of dust mass growth. Thus, the critical metallicity of Inoue (2011) is the metallicity at which the contribution of dust mass growth exceeds that of dust destruction. In contrast, our critical metallicity is the metallicity at which the dust mass growth becomes the main source of the increase of dust (the contribution of dust mass ejected by stars is the main source of dust at an early stage of galaxy evolution). Those interested in both works should bear this difference in mind.

In Fig. 3, we show the relation between the metallicity and the dust-to-gas mass ratio (the left panel is normalized by the critical metallicity, whilst the right panel is not) for $\tau_{\mathrm{SF}}=0.5,5,50$ Gyr. In Fig. 3, we find different evolutionary tracks depending on $\tau_{\mathrm{SF}}$ in the right-hand panel, whereas these tracks, with the metallicity normalized to the critical metallicity, are well overlaid on each other in the left-hand panel. This clearly illustrates the significance of the critical metallicity. The dust mass growth becomes dominant not when the galactic age reaches a certain value, but when the metallicity exceeds the critical value. In the left panel, the dust-to-gas mass ratio increases rapidly after metallicity exceeds the point of the critical metallicity. Thus, dust produced by dust mass growth becomes the dominant contribution to the dust mass of a galaxy if $Z>Z_{\text {cr }}$ in the galaxy.

In Fig. 4, we show the critical metallicity as a function of $\tau_{\mathrm{SF}}$. As shown in the figure, the critical metallicity becomes larger if the star-formation timescale is shorter. As mentioned in Section 1 and 3.1, the dust mass growth is expected to be the dominant source of dust in various galaxies (e.g., the Milky Way: Draine (2009) and dusty QSOs at high redshifts ${ }^{2}$ : Valiante et al. (2011, among others), in spite of different star formation timescales and ages of the galaxies. In terms of the critical metallicity, we can explain the reason 

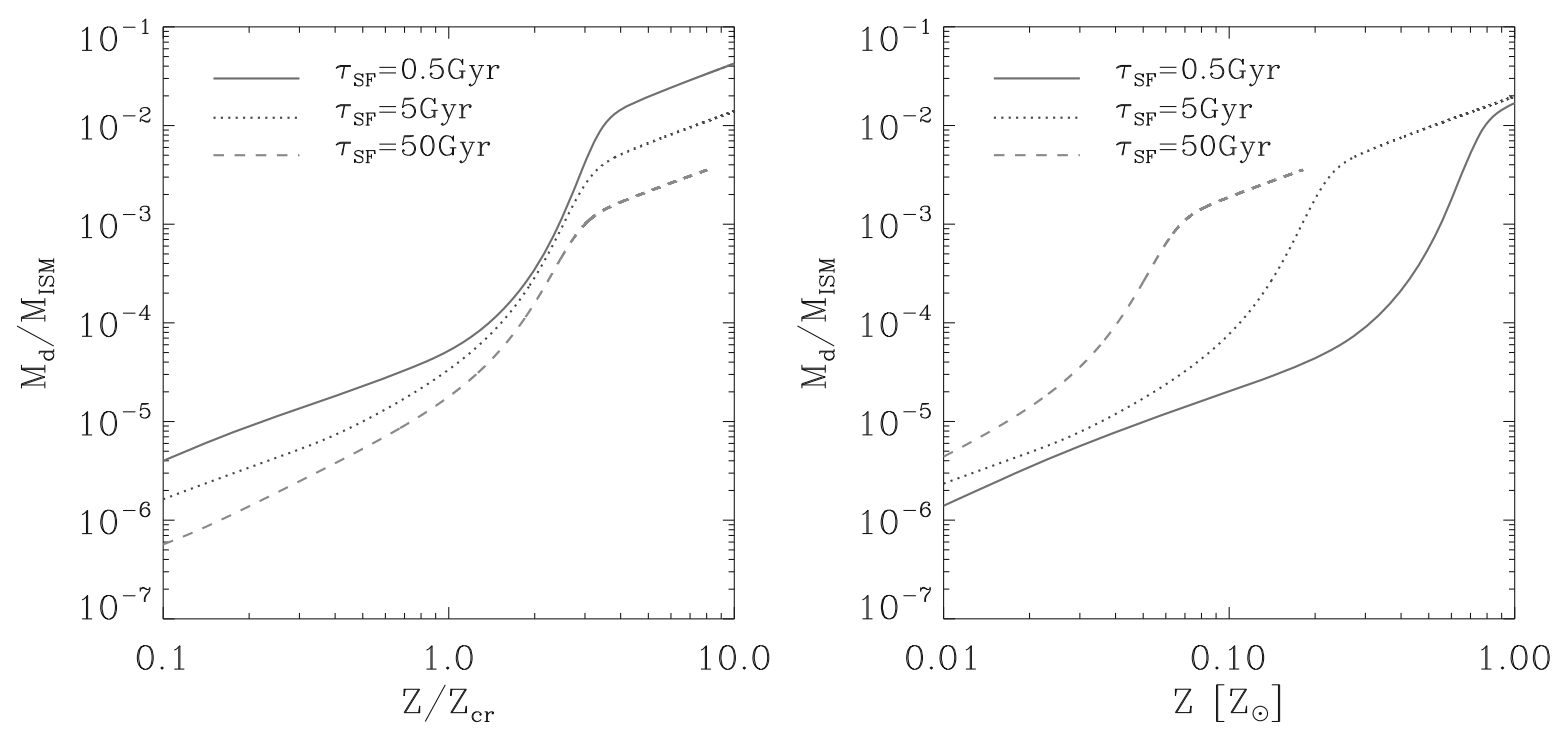

Fig. 3. The metallicity—dust-to-gas mass ratio with $\eta=1.00$ and $M_{\mathrm{tot}}=10^{10} \mathrm{M}_{\odot}$. Left panel: normalized by the critical metallicity. Right panel: not normalized. Solid, dotted and dashed lines represent $\tau_{\mathrm{SF}}=0.5,5,50 \mathrm{Gyr}$, respectively. Dashed and solid horizontal lines represent $\beta=1.0$ and 10.0 , respectively.

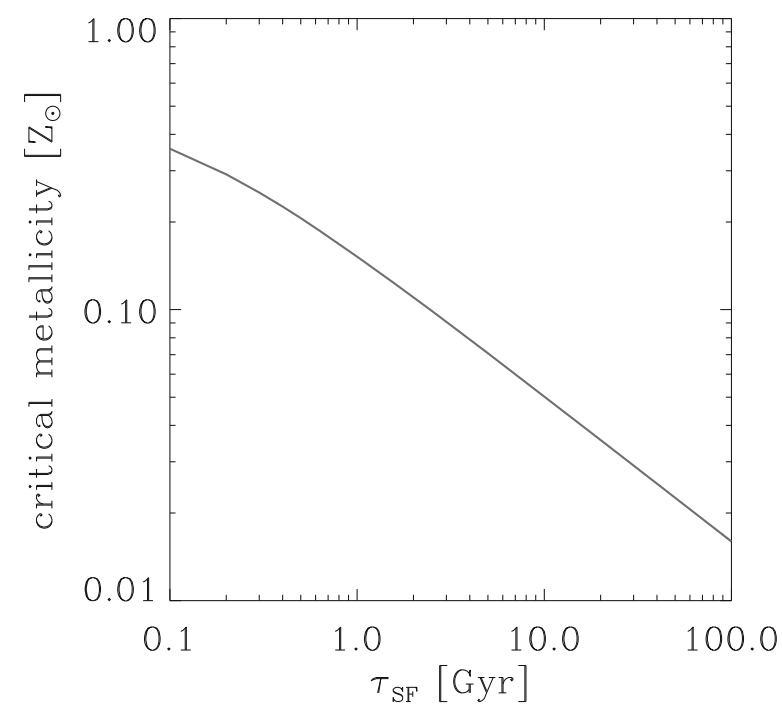

Fig. 4. Critical metallicity as a function of $\tau_{\mathrm{SF}}$ with $\eta=1.00$.

for this in a coherent manner: the metallicity in the galaxies exceeds the critical metallicity.

Here, we emphasize the importance of the metallicity dependence in the accretion growth timescale, $\tau_{\text {acc }}$. Pipino et al. (2011) argued that the dust mass growth was important to explain the observed huge mass of dust in high-z QSOs. However, they did not seem to consider the metallicity dependence in $\tau_{\mathrm{acc}}{ }^{3}$. Figure 5 shows the effect of the metallicity dependence on the dust mass evolution. When we consider the dependence properly, the point where the dust mass growth becomes dominant is delayed until the metallicity exceeds the critical value as discussed above. On the other hand, if we omit the dependence, and take a constant

${ }^{2}$ It is considered that QSOs in a high- $z$ Universe have a high star-formation rate and even and larger subsolar metallicities (e.g., Juarez et al., 2009; Matsuoka et al., 2009).

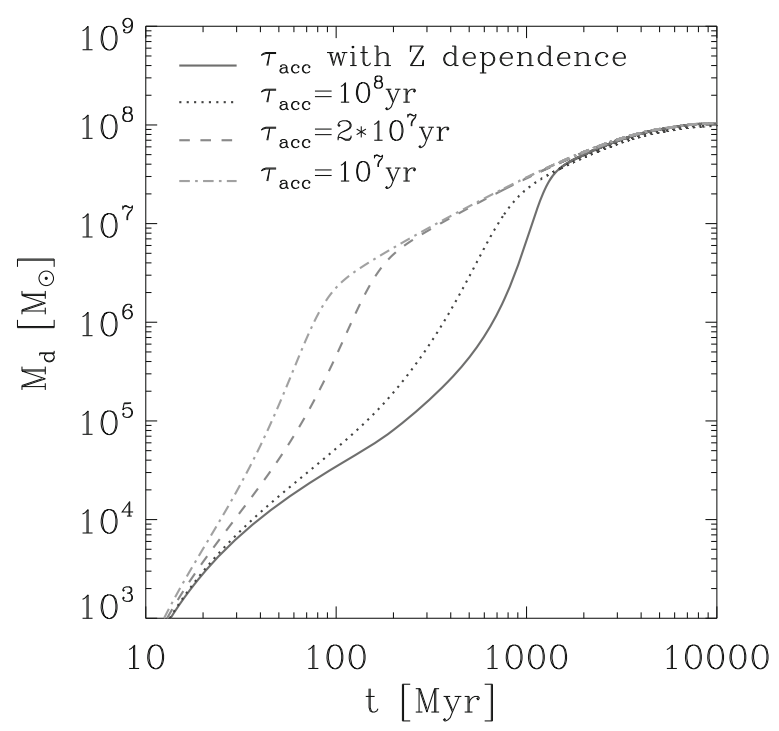

Fig. 5. Time evolution of the total dust mass with (solid line) and without (other lines) metallicity dependence for the dust mass growth timescale. Here we adopt $\tau_{\mathrm{SF}}=5 \mathrm{Gyr}, M_{\mathrm{tot}}=10^{10} \mathrm{M}_{\odot}$ and $\eta=1.00$. The three values of $\tau_{\text {acc }}$ represent $Z=0.2 Z_{\odot}$ (dotted line), $Z=1.0 Z_{\odot}$ (dashed line) and $Z=2.0 Z_{\odot}$ (dot-dashed line), respectively.

value for $\tau_{\text {acc }}$, the point when the growth becomes the dominant contribution to the total dust mass is determined by just the adopted $\tau_{\text {acc }}$.

In the above discussion, we have focused on the critical metallicity. One may, however, be interested in its relation to the time, $t_{\mathrm{cr}}$, which is the galactic age when the metallicity in a galaxy reaches the critical metallicity. Here, in order to understand, more clearly, the importance of the dust mass growth in various galaxies with various star-formation

${ }^{3}$ Indeed, their equations (4) and (5) have the metallicity dependence. However, their adopted timescales in table 1 seem to omit the dependence finally. 


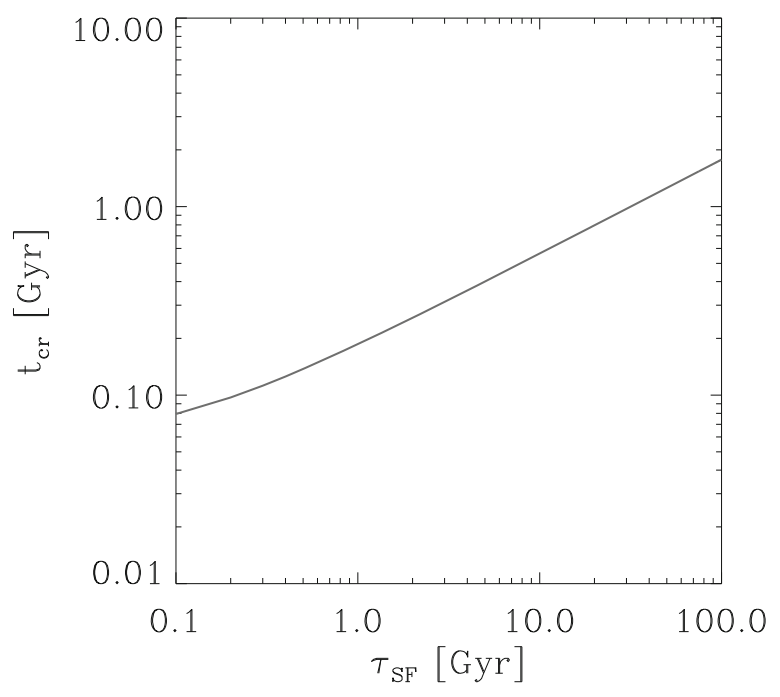

Fig. 6. $t_{\mathrm{cr}}$ as a function of $\tau_{\mathrm{SF}}$ with $\eta=1.00$.

timescales, we demonstrate the relation between the critical metallicity $Z_{\mathrm{cr}}$ and the time $t_{\mathrm{cr}}$. However, we stress that the metallicity is more fundamental because $t_{\mathrm{cr}}$ is determined by the critical metallicity.

We derive the relation using Eqs. (2) and (3). If $A$ is defined as

$$
\int_{m_{\text {cut }}(t)}^{100 \mathrm{M}_{\odot}} m_{Z}\left(m, Z\left(t-\tau_{m}\right)\right) \phi(m) \mathrm{d} m \equiv A,
$$

then, the relation is expressed as

$$
t_{\mathrm{cr}}=\frac{\tau_{\mathrm{SF}}}{A} Z_{\mathrm{cr}}
$$

where $A$ is a constant, and is about 0.018 in our calculation. If an inflow process occurs, since the dust-to-gas mass ratio and metallicity become smaller than the case without inflow, it is sufficient to consider the larger $\tau_{\mathrm{SF}}$. Figure 6 shows $t_{\mathrm{cr}}$ as a function of $\tau_{\mathrm{SF}}$. From this figure, we find that $t_{\mathrm{cr}}$ becomes shorter if $\tau_{\mathrm{SF}}$ is shorter, while in the case of $Z_{\mathrm{cr}}$, the trend is the opposite (Fig. 4). This is explained by the following reason. If the $\tau_{\mathrm{SF}}$ is short, the fast progress of the star formation causes the metallicity to become large at an early stage of galaxy evolution. Hence, although the critical metallicity is large in the case with short $\tau_{\mathrm{SF}}, t_{\mathrm{cr}}$ is short due to the fast evolution of metallicity. From the relation between $z_{\mathrm{cr}}$ and $t_{\mathrm{cr}}$, we can understand that the dust mass growth is the main dust production of the Milky Way (evolved galaxy) and dusty QSOs (in a high-z Universe).

Next, we discuss the effect of the grain size distribution. Recently, Hirashita and Kuo (2011) showed that the dust mass growth in the ISM depends on the grain size distribution of a galaxy. To examine the effect of the grain size on the dust mass growth, we consider the case with $\bar{a}=0.01$ $\mu \mathrm{m}$ (the case with $\bar{a}=0.1 \mu \mathrm{m}$ is the fiducial case in this paper). Figure 7 shows the relation between the metallicity and the dust-to-gas mass ratio for $\bar{a}=0.1 \mu \mathrm{m}$ (solid line) and $0.01 \mu \mathrm{m}$ (dotted line) with $\tau_{\mathrm{SF}}=5 \mathrm{Gyr}$ and $\eta=1.00$. From Eqs. (20) and (24), the critical metallicity as a function of $\bar{a}$ is expressed as

$$
Z_{\mathrm{cr}}(\bar{a})=(\bar{a} / 0.1 \mu \mathrm{m})^{1 / 2} \mathrm{Z}_{\mathrm{cr}}(0.1 \mu \mathrm{m}) .
$$

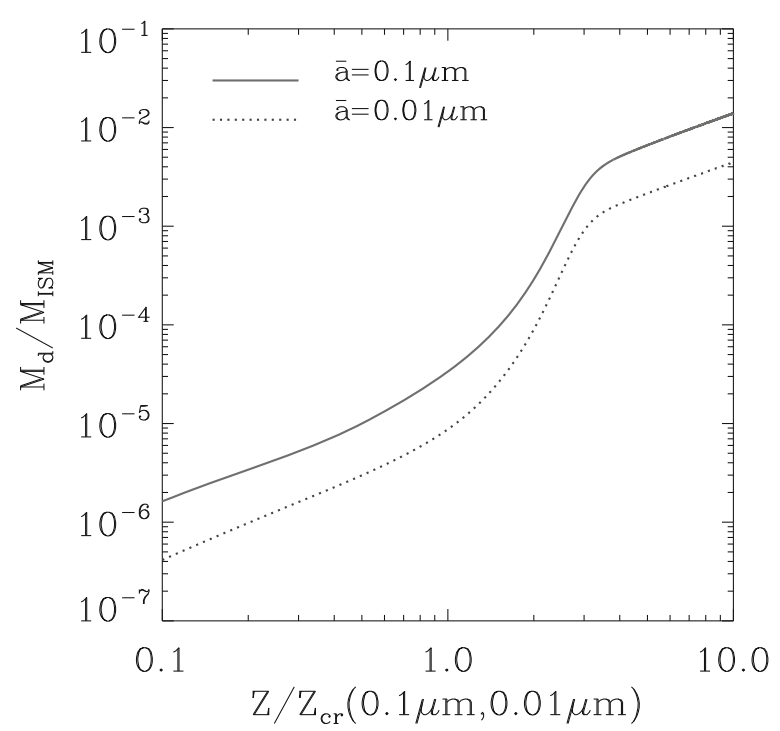

Fig. 7. The relation between metallicity and dust-to-gas mass ratio for $\bar{a}=0.1 \mu \mathrm{m}$ (solid line) and $0.01 \mu \mathrm{m}$ (dotted line) with $\tau_{\mathrm{SF}}=5 \mathrm{Gyr}$ and $\eta=1.00$. From Eq. $(27), Z_{\mathrm{cr}}(0.01 \mu \mathrm{m}) \sim 0.32 Z_{\mathrm{cr}}(0.1 \mu \mathrm{m})$.

From Fig. 7, we find that the evolutionary tracks with different $\bar{a}$ show almost the same behavior if we introduce the critical metallicity for each value of $\bar{a}$. Thus, although Hirashita and Kuo (2011) showed that the critical metallicity is sensitive to the grain size distribution, the mechanism that the critical metallicity determines the point at which the dust mass growth becomes the dominant factor in the growth of the total dust mass in a galaxy does not change. As for the dust evolution considered the evolution of the grain size distribution (including the effects of stellar dust, SN destruction and accretion) in a galaxy, this issue will be extensively discussed in a work in preparation (Asano et al. 2012, in preparation).

\section{Conclusions}

In this work, we have constructed a galaxy evolution model taking into account the metallicity and age dependence on the various dust sources (AGB stars, SNe II and growth in the ISM) to investigate what is the main driver of the grain growth which is expected to be the dominant source of dust in various galaxies with various starformation timescales.

We have found that the point at which the dust mass growth in the ISM becomes dominant is determined by the metallicity. If the metallicity in a galaxy exceeds a certain critical value, the critical metallicity, dust mass growth becomes active and the dust mass rapidly increases, until metals are depleted from the ISM. This critical metallicity is larger for a shorter star-formation timescale. Dust mass growth is thought to be the dominant source of dust in evolved galaxies, such as the Milky Way and young, but dusty and massive, QSOs at high redshifts. The importance of the dust mass growth in such a diversity of galaxies can be explained clearly in terms of the critical metallicity. The dust mass growth in the ISM is regulated by the metallicity, and we emphasize that the critical metallicity works as an indicator to judge whether the grain growth in the ISM is 
the dominant source of dust in a galaxy, especially because of a strong and nonlinear dependence on the metallicity.

Acknowledgments. We thank the anonymous referees for their helpful comments which improved the presentation and content of this paper. We are grateful to Takashi Kozasa, Takaya Nozawa, Daisuke Yamasawa, Asao Habe, and Takako T. Ishii for fruitful discussions. RSA has been supported from the Grant-in-Aid for JSPS Research under Grant No. 23-5514. RSA and TTT have been also partially supported from the Grant-in-Aid for the Global COE Program "Quest for Fundamental Principles in the Universe: from Particles to the Solar System and the Cosmos" from the Ministry of Education, Culture, Sports, Science and Technology (MEXT) of Japan. TTT and AKI have been supported by Program for Improvement of Research Environment for Young Researchers from Special Coordination Funds for Promoting Science and Technology, and the Grant-in-Aid for the Scientific Research Fund (TTT: 20740105, 23340046, AKI: 19740108) commissioned by the MEXT. HH is supported by NSC grant 99-2112-M-001006-MY3.

\section{Appendix A. What Dominates $\delta$ Before the Dust Mass Growth Becomes Effective?}

Figure A.1 shows the time evolution of the ratio of dust mass produced by stars to metal mass ejected by stars, $\delta_{\text {star }}$ (thick lines) and $\delta$ (thin lines) with $\tau_{\mathrm{SF}}=0.5,5,50 \mathrm{Gyr}$. From Eqs. (7)-(9), the ratio $\delta_{\text {star }}$ is expressed as

$$
\delta_{\mathrm{star}}(t)=\frac{Y_{\mathrm{d}}(t)}{R_{Z}(t)+Y_{Z}(t)}
$$

From Fig. A.1, we observe that the evolutionary tracks of $\delta_{\text {star }}$ are almost the same tracks before the dust mass growth dominates the total dust mass production in galaxies. Hence, $\delta$ before the dust mass growth becomes dominant is determined by $\delta_{\text {star }}$.

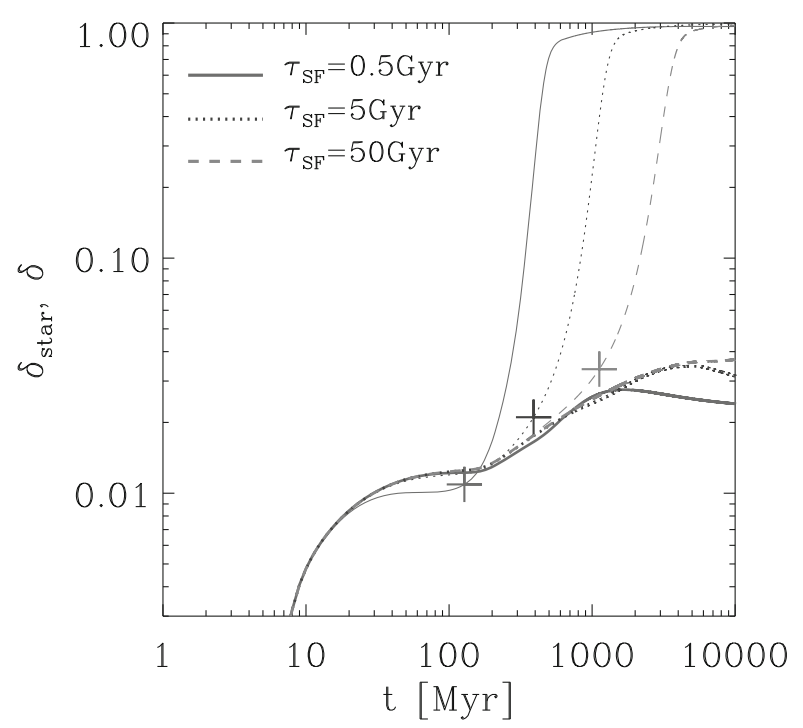

Fig. A.1. Time evolution of the ratio of dust mass produced by stars to metal mass ejected by stars. Solid, dotted and dashed lines represent $\tau_{\mathrm{SF}}=0.5,5,50 \mathrm{Gyr}$, respectively. For comparison, the results of Fig. 2 with thin lines are overlaid on the panel. Cross symbols mark the switching point for each $\tau_{\mathrm{SF}}$.

\section{References}

Anders, E. and N. Grevesse, Abundances of the elements-Meteoritic and solar, Geochim. Cosmochim. Acta., 53, 197-214, 1989.

Beelen, A., P. Cox, D. J. Benford, C. D. Dowell, A. Kovács, F. Bertoldi, A. Omont, and C. L. Carilli, $350 \mu \mathrm{m}$ dust emission from high-redshift quasars, Astrophys. J., 642, 694-701, 2006.

Bianchi, S. and R. Schneider, Dust formation and survival in supernova ejecta, Mon. Not. R. Astron. Soc., 378, 973-982, 2007.

Calura, F., A. Pipino, and F. Matteucci, The cycle of interstellar dust in galaxies of different morphological types, Astron. Astrophys., 479, 669$85,2008$.

Draine, B. T., Interstellar dust models and evolutionary implications, in Cosmic Dust-Near and Far, edited by T. Henning, E. Grün, and J. Steinacker, 20 pp., Astronomical Society of the Pacific, San Francisco, 2009.

Dwek, E., The Evolution of the elemental abundances in the gas and dust phases of the galaxy, Astrophys. J., 501, 643-665, 1998.

Dwek, E. and J. M. Scalo, The evolution of refractory interstellar grains in the solar neighborhood, Astrophys. J., 239, 193-211, 1980.

Gall, C., A. C. Andersen, and J. Hjorth, Genesis and evolution of dust in galaxies in the early Universe. I. Modelling dust evolution in starburst galaxies, Astron. Astrophys., 528, A13, 2011a.

Gall, C., A. C. Andersen, and J. Hjorth, Genesis and evolution of dust in galaxies in the early Universe. II. Rapid dust evolution in quasars at $z \gtrsim 6$, Astron. Astrophys., 528, A14, 2011 b.

Granato, G. L., C. G. Lacey, L. Silva, A. Bressan, C. M. Baugh, S. Cole, and C. S. Frenk, The infrared side of galaxy formation. I. The local universe in the semianalytical framework, Astrophys. J., 542, 710-730, 2000.

Heger, A., C. L. Fryer, S. E. Woosley, N. Langer, and D. H. Hartmann, How massive single stars end their life, Astrophys. J., 591, 288-300, 2003.

Hirashita, H., Global law for the dust-to-gas ratio of spiral galaxies, Astrophys. J., 510, L99-L102, 1999a.

Hirashita, H., Dust-to-gas ratio and metallicity in dwarf galaxies, Astrophys. J., 522, 220-224, 1999b.

Hirashita, H., Cyclic changes in dust-to-gas ratio, Astrophys. J., 531, 693$700,2000$.

Hirashita, H. and A. Ferrara, Effects of dust grains on early galaxy evolution, Mon. Not. R. Astron. Soc., 337, 921-937, 2002.

Hirashita, H. and T.-M. Kuo, Effects of grain size distribution on the interstellar dust mass growth, Mon. Not. R. Astron. Soc., 416, 13401353, 2011.

Hirashita, H. and H. Yan, Shattering and coagulation of dust grains in interstellar turbulence, Mon. Not. R. Astron. Soc., 394, 1061-1074, 2009.

Hollenbach, D. and C. F. McKee, Molecule formation and infrared emission in fast interstellar shocks. I Physical processes, Astrophys. J. S., 41, 555-592, 1979.

Inoue, A. K., Evolution of dust-to-metal ratio in galaxies, Publ. Astron. Soc. Jpn., 55, 901-909, 2003.

Inoue, A. K., The origin of dust in galaxies revisited: the mechanism determining dust content, Earth Planets Space, 63, 1027-1039, 2011.

Jones, A. P. and J. A. Nuth, III, Dust destruction in the ISM: A reevaluation of dust lifetimes, Astron. Astrophys., 530, A44, 2011.

Jones, A. P., A. G. G. M. Tielens, D. J. Hollenbach, and C. F. McKee, Grain destruction in shocks in the interstellar medium, Astrophys. J., 433, 797-810, 1994.

Jones, A. P., A. G. G. M. Tielens, and D. J. Hollenbach, Grain shattering in shocks: The interstellar grain size distribution, Astron. J., 469, 740-764, 1996.

Juarez, Y., R. Maiolino, R. Mujica, M. Pedani, S. Marinoni, T. Nagao, A. Marconi, and E. Oliva, The metallicity of the most distant quasars, Astron. Astrophys., 494, L25-L28, 2009.

Larson, R. B., Early star formation and the evolution of the stellar initial mass function in galaxies, Mon. Not. R. Astron. Soc., 301, 569-581, 1998.

Liang, S. L. and A. Li, Proving cosmic dust of the early universe through high-redshift gamma-ray bursts, Astrophys. J. L., 690, L56-L60, 2009.

Liffman, K. and D. D. Clayton, Stochastic evolution of refractory interstellar dust during the chemical evolution of a two-phase interstellar medium, Astrophys. J., 340, 853-868, 1989.

Lisenfeld, U. and A. Ferrara, Dust-to-gas ratio and metal abundance in dwarf galaxies, Astron. J., 496, 145-154, 1998.

Mathis, J. S., W. Rumpl, and K. H. Nordsieck, The size distribution of interstellar grains, Astrophys. J., 217, 425-433, 1977. 
Matsuoka, K., T. Nagao, R. Maiolino, A. Marconi, and Y. Taniguchi, Chemical evolution of high-redshift radio galaxies, Astron. Astrophys., 503, 721-730, 2009.

Matteucci, F., E. Spitoni, S. Recchi, and R. Valiante, The effect of different type Ia supernova progenitors on Galactic chemical evolution, Astron. Astrophys., 501, 531-538, 2009.

McKee, C. F., Dust destruction in the interstellar medium, in Interstellar Dust, edited by L. Allamandora and A. G. G. M. Tielens, 14 pp., Kluwer Academic Publishers, Dordrecht, 1989.

Michałowski, M. J., E. J. Murphy, J. Hjorth, D. Watson, C. Gall, and J. S. Dunlop, Dust grain growth in the interstellar medium of $5<z<6.5$ quasars, Astron. Astrophys., 522, A15, 2010a.

Michałowski, E. J., D. Watson, and J. Hjorth, Rapid dust production in submillimeter galaxies at $z>4$ ?, Astrophys. J., 712, 942-950, 2010 b.

Noll, S., D. Burgarella, E. Giovannoli, V. Buat, D. Marcillac, and J. C. Muñoz-Mateos, Analysis of galaxy spectral energy distributions from far-UV to far-IR with CIGALE: Studying a SINGS test sample, Astron. Astrophys., 507, 1793-1813, 2009

Nozawa, T., T. Kozasa, H. Umeda, K. Maeda, and K. Nomoto, Dust in the early universe: Dust formation in the ejecta of population III supernovae, Astrophys. J., 598, 785-803, 2003.

Nozawa, T., T. Kozasa, and A. Habe, Dust destruction in the high-velocity shocks driven by supernovae in the early universe, Astron. J., 648, 435451,2006

Nozawa, T., T. Kozasa, A. Habe, E. Dwek, H. Umeda, N. Tominaga, K. Maeda, and K. Nomoto, Evolution of dust in primordial supernova remnants: Can dust grains formed in the ejecta survive and be injected into the early interstellar medium?, Astrophys. J., 666, 955-966, 2007.

Nozawa, T., T. Kozasa, N. Tominaga, K. Maeda, H. Umeda, K. Nomoto, and $\mathrm{O}$. Krause, Formation and evolution of dust in type IIb supernovae with application to the cassiopeia a supernova remnant, Astrophys. J., 713, 356-373, 2010

Nozawa, T., K. Maeda, T. Kozasa, M. Tanaka, K. Nomoto, and H. Umeda, Formation of dust in the ejecta of type Ia supernovae, Astrophys. J., 736, 45-57, 2011

Pipino, A., X. L. Fan, F. Matteucci, F. Calura, L. Silva, G. Granato, and R. Maiolino, The chemical evolution of elliptical galaxies with stellar and QSO dust production, Astron. Astrophys., 525, A61, 2011.

Popescu, C. C., R. J. Tuffs, M. A. Dopita, J. Fischera, N. D. Kylafis, and
B. F. Madore, Modelling the spectral energy distribution of galaxies. V. The dust and PAH emission SEDs of disk galaxies, Astron. Astrophys., 527, A109, 2011.

Raiteri, C. M., M. Villata, and J. F. Navarro, Simulations of Galactic chemical evolution. I. O and Fe abundances in a simple collapse model, Astron. Astrophys., 315, 105-115, 1996.

Schmidt, M., The rate of star formation, Astrophys. J., 129, 243-258, 1959. Spitzer, L., Jr., Physical properties of grains, in Physical Processes in the Interstellar Medium, 23 pp., Wiley, New York, 1978.

Takagi, T., N. Arimoto, and V. Vansevičius, Age and dust degeneracy for starburst galaxies solved?, Astrophys. J., 523, 107-113, 1999.

Valiante, R., R. Schneider, S. Bianchi, and A. C. Andersen, Stellar sources of dust in the high-redshift Universe, Mon. Not. R. Astron. Soc., 397, 1661-1671, 2009.

Valiante, R., R. Schneider, S. Salvadori, and S. Bianchi, The origin of the dust in high-redshift quasars: The case of SDSS J1148+5251, Mon. Not. R. Astron. Soc., 416, 1916-1935, 2011.

van den Hoek, L. B. and M. A. T. Groenewegen, New theoretical yields of intermediate mass stars, Astron. Astrophys. S., 123, 305-328, 1997.

Wang, R., J. Wagg, C. L. Carilli, D. J. Benford, C. D. Dowell, F. Bertoldi, F. Walter, K. M. Menten, A. Omont, P. Cox, M. A. Strauss, X. Fan, and L. Jiang, SHARC-II $350 \mu \mathrm{m}$ observations of thermal emission from warm dust in $z \geqslant 5$ quasars, Astron. J., 135, 1201-1206, 2008.

Witt, A. N. and K. D. Gordon, Multiple scattering in clumpy media. II. Galactic environments, Astrophys. J., 528, 799-816, 2000.

Woosley, S. E. and T. A. Weaver, The evolution and explosion of massive stars. II. Explosive hydrodynamics and nucleosynthesis, Astrophys. J. S., 101, 181-235, 1995.

Yamasawa, D., A. Habe, T. Kozasa, T. Nozawa, H. Hirashita, H. Umeda, and K. Nomoto, The role of dust in the early universe. I. Protogalaxy evolution, Astrophys. J., 735, 44-57, 2011.

Zhukovska, S., H. P. Gail, and M. Trieloff, Evolution of interstellar dust and stardust in the solar neighbourhood, Astron. Astrophys., 479, 453480, 2008.

R. S. Asano (e-mail: asano.ryosuke@g.mbox.nagoya-u.ac.jp), T. T Takeuchi, H. Hirashita, and A. K. Inoue 